\title{
A mutant mouse menagerie
}

\author{
Geneticists are set to be the winners in a chemical lottery, as a mammoth \\ range of randomly mutated mice promises them off-the-shelf tools for \\ defining gene function. Alison Abbott investigates.
}

A $\mathrm{n}$ army of mutant mice is poised to invade the laboratories of geneticists. This month's Nature Genetics ${ }^{1,2}$ reports the results from two new screens for these rodents, which should make it easier to use the mouse genome as a guide to deciphering our own genetic blueprint.

When trying to determine the function of a given gene, geneticists often use 'knockout' mice. This involves selecting and disabling a particular gene in the mice in an attempt to discover what it does.

The new screens are based on chemical mutagenesis which, by comparison, seems relatively crude. Mutants are generated randomly so that, for example, immunogeneticists can use those mice with specific immune deficiencies to look for genes involved in the development of the immune system, and neurologists can study the mutants with defective nervous systems.

When the idea of mouse mutagenesis factories was mooted at the International Mouse Genome Conference in London in 1994, many were sceptical of this phenotypedriven approach. But Rudi Balling and Steve Brown were undeterred, and three years later they opened the world's first large mutantmouse screens - Balling at the GSF National Research Centre for Environment and Health in Munich, and Brown at the Medical Research Council's Mammalian Genetics Unit in Harwell, Oxfordshire. The two facilities have now produced some 700 strains of mutant mice with traits including deafness, behavioural abnormalities and metabolic disorders, and this figure is set to rise by several hundred mutants per year.

\section{A timely result}

The arrival of these mutants is timely. The mouse genome should be sequenced within the next two years, and geneticists hope that mutagenesis screens will help them to assign functions to mouse genes - and hence to their human counterparts.

The new mutants will soon be followed by others. Similar screens were launched in 1998 at the Australian National University in Canberra, and this year at the RIKEN Genomic Sciences Center in Tsukuba, Japan. In the United States, two screens for neurological mutants will this month get funding from the National Institutes of Health.

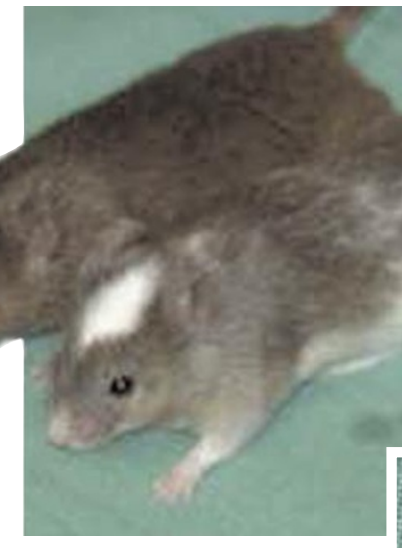

In all the screens, male mice are injected with ethylnitrosourea, a chemical that induces random mutations across the genome of cells, including sperm. The males are then mated with normal females, and bred through several generations. Similar techniques have helped unravel the genetics of the fruitfly Drosophila $^{3}$, the nematode Caenorhabditis elegans ${ }^{4}$ and the zebra fish Danio rerio ${ }^{5,6}$.

The difficult part is screening thousands of animals to identify those that carry interesting mutations. At Harwell, mice are put through an initial suite of 30 or 40 tests, such as observing their gait, which takes an experienced technician around 15 minutes per mouse. Other tests involve biological assays. For example, Klaus Pfeffer of the Technical University of Munich has designed a battery of 60 immunological tests for the GSF.

\section{Dominant position}

Most mutants described in the new papers are dominant. "We are pleased that the dominant screens have thrown up such a variety of interesting mutants," says Brown. But looking for recessive mutants, which emerge in subsequent generations, will be important. In the Drosophila research, recessive mutants outnumbered dominant ones by a factor of five. In the mouse, such mutants may provide valuable models for medical research. "Many diseases in humans are recessive," says Martin Hrabé de Angelis, the current director of the GSF screen.

In the long term, the mutation factories might investigate interactions between genes and create models for complex, multigene diseases. This would be done by crossing mutants with inbred strains of mice that have
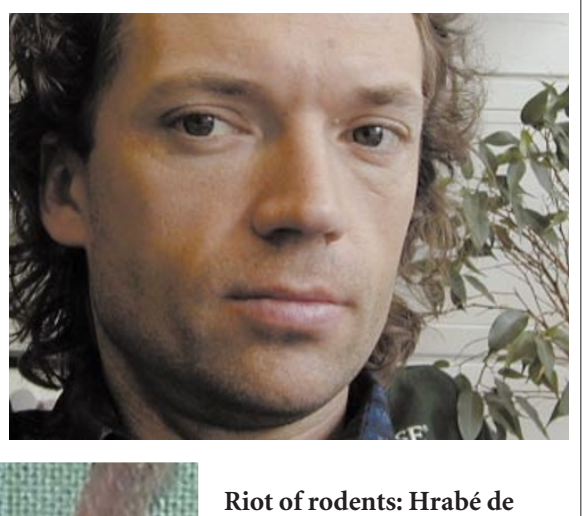
Angelis directs one of the two screens generating randomly mutated mice for genetic analysis. different genetic backgrounds. Already, researchers at the GSF are studying geneenvironment interactions. For example, they are identifying mutants that struggle to repair radiation damage to their DNA. "This may eventually allow us to identify patients who would suffer extreme side effects from radiation therapy," says Hrabé de Angelis.

Biologists have already started using mutants from the two screens. Ian Jackson of the Medical Research Council's Human Genetics Unit in Edinburgh is studying mice with mutations in the gene for the enzyme phosphodiesterase B. Defects in this enzyme cause autosomal recessive retinal pigmentosis, a hereditary disease that results in progressive blindness. "We have several mice with different mutations in the same gene, and each mutant displays a different severity of phenotype," says Jackson. Pfeffer is similarly pleased to have identified mutants with autoimmunity, which might yield insights into rheumatoid arthritis.

Peter Gruss, a leader in knockout-mouse research at the Max Planck Institute for Biophysical Chemistry in Göttingen, says that the screens have already proved their worth. "But gene-driven and phenotypedriven approaches are complementary and both are needed," he adds.

Alison Abbott is Nature's Senior European Correspondent.

1. Nolen, P. M. et al. Nature Genet. 25, 440-443 (2000).

2. Hrabé de Angelis, M. et al. Nature Genet. 25, 444-447 (2000).

3. Nüsslein-Volhard, C. \& Wieshaus, E. Nature 287, 795-801

(1980)

4. Brenner, S. Genetics 77, 71-94 (1974).

5. Haffter, P. et al. Development 123, 1-36 (1996).

6. Driever, W. et al. Development 123, 37-46 (1996). 